Obere Extremität 2022 $17: 27-33$ https://doi.org/10.1007/s11678-021-00674-5

Eingegangen: 27. August 2021

Angenommen: 29. November 2021

Online publiziert: 23. Dezember 2021

(c) Der/die Autor(en) 2021

\section{Quantifizierung der Belastungen des Muskel-Band-Apparats im Schultergelenk von Speerwerfern}

\author{
Hans-Peter Köhler' (D) $\cdot$ Pierre Hepp ${ }^{2} \cdot$ Maren Witt $^{1}$ \\ 'Sportwissenschaftliche Fakultät, Abteilung Sportbiomechanik, Universität Leipzig, Leipzig, Deutschland \\ ${ }^{2}$ Klinik und Poliklinik für Orthopädie, Unfallchirurgie und Plastische Chirurgie, Universitätsklinikum \\ Leipzig, Leipzig, Deutschland
}

\title{
Zusammenfassung
}

Hintergrund: Verletzungen der Schulter sind häufige Ursachen für den Verlust von Trainingszeit und Wettkampfpraxis. Dabei sind sowohl akute Verletzungen als auch langfristige Veränderungen der stabilisierenden Strukturen problematisch. Während über die Belastungssituation der Schulter im Baseball bereits einige Studien vorliegen, ist dies im Speerwurf bisher nicht der Fall, weder die Kinematik noch die Kinetik wurden bisher vollständig quantifiziert.

Ziel der Arbeit: Das Ziel der Arbeit bestand darin, die Belastung der Schulter im Speerwurf zu quantifizieren und somit einen Beitrag zu Identifikation von Verletzungsrisiken zu leisten.

Material und Methoden: Von 10 männlichen Speerwerfern wurden mithilfe eines Infrarotkamerasystems und 18 reflektierenden Markern an prominenten Körperpunkten die Bewegungsdaten erfasst. Diese Daten dienten unter Nutzung eines 5-segmentigen Mehrkörpermodells dazu, die Kinematik und Kinetik des Schultergelenks zu modellieren. Aus den berechneten Verläufen wurden jeweils die Maxima extrahiert und einer Zusammenhangsprüfung zur Abwurfgeschwindigkeit unterzogen.

Ergebnisse: Die Sportler erreichten eine mittlere Abwurfgeschwindigkeit von $23,29 \pm 2,17 \mathrm{~ms}^{-1}$. Die maximalen Gelenkwinkelgeschwindigkeiten lagen zwischen $445-4071 \%$ in Abhängigkeit von der Bewegungsebene. Auch in den Gelenkbelastungen zeigten sich spezifische Unterschiede in den Ebenen (109-129 Nm).

Schlussfolgerung: Die Belastungssituation im Speerwurf unterscheidet sich im Vergleich zum Baseball. Während im Speerwurf verringerte Amplituden und Winkelgeschwindigkeiten auftreten, wird eine erhöhte Kraftanforderung sichtbar. Als mögliche Ursachen hierfür werden unterschiedliche Regelwerke diskutiert (Gerätegewicht/-dimensionen, Eingangsgeschwindigkeit).

\section{Schlüsselwörter}

Rotatorenmanschette $\cdot$ Dynamik $\cdot$ Modellierung $\cdot$ Simulation $\cdot$ Leistungssport

\section{Hintergrund}

Verletzungen der oberen Extremitäten sind in Wurfsportarten einer der häufigsten Ausfallgründe. Während im Baseball Schulterverletzungen mit 12-19\% die häufigste Verletzungsursache ausmachen $[10,26]$, beträgt der Anteil im Handball noch 9,8\%, wobei diese Sportler überdurchschnittlich oft durch lange Ausfallzeiten (> 21 Tage) betroffen sind [18]. Für die leichtathletischen Würfe bzw. den Speerwurf sind zwar die unteren Extremitäten, wie auch im Handball, häufiger von Verletzungen betroffen, jedoch entfallen ca. $7 \%$ der Verletzungen auf das Schultergelenk [5]. Langfristig erleiden ca. $75 \%$ der Werfer im Karriereverlauf eine oder mehrere akute Schulterverletzungen, wobei es sich bei $40 \%$ um schwere Verletzungen (Ausfallzeit $>28$ Tage) handelt [7]. Durch die langjährige, hoch intensive Belastung des 


\begin{tabular}{|c|c|c|}
\hline Verletzung & Mechanismus & MRT morphologische Veränderungen \\
\hline \multicolumn{3}{|c|}{ Häufigsten Verletzungen } \\
\hline GIRD & Chronisches Einreißen der hinteren Kapsel durch wiederholtes Werfen & $\begin{array}{l}\text { Posterior/posteriorinferiore Kapselverdickung und } \\
\text {-verkürzung }\end{array}$ \\
\hline PSI & $\begin{array}{l}\text { Kontakt der Unterseite der Rotatorenmanschette zwischen Tuberculum majus } \\
\text { und posterosuperiorem Glenoid bzw. Labrum in der Abduktionsaußenrotation }\end{array}$ & $\begin{array}{l}\text { Strukturelle Veränderungen des dorsalen Hume- } \\
\text { ruskopfes, subkortikale Zystenbildung, Knochen- } \\
\text { marködem („,bone bruise"), kortikale Abflachung }\end{array}$ \\
\hline SLAP & $\begin{array}{l}\text { Übermäßige Außenrotation „schält” den Bizeps-Labrum-Komplex unter Belas- } \\
\text { tung }\end{array}$ & $\begin{array}{l}\text { Am häufigsten: Ablösung des Bizepsankers und } \\
\text { des hinteren Labrums vom Glenoid (Typ II) }\end{array}$ \\
\hline $\begin{array}{l}\text { Partielle } \\
\text { Rotatoren- } \\
\text { manschet- } \\
\text { teneinrisse }\end{array}$ & $\begin{array}{l}\text { Akute Spannungsüberlastung oder chronisches Repetitionstrauma; internes } \\
\text { Impingement führt zu einer Kompressionsverletzung, übermäßige Außenro- } \\
\text { tation führt zu einer Torsionsverletzung der Sehnen der Rotatorenmanschette }\end{array}$ & $\begin{array}{l}\text { Gelenkseitige Fasern der hinteren Anteile des } \\
\text { M. supraspinatus und des vorderen M. infraspina- } \\
\text { tus }\end{array}$ \\
\hline \multicolumn{3}{|c|}{ Verletzungen der ventralen Schulteranteile } \\
\hline $\begin{array}{l}\text { Vordere } \\
\text { Kapselver- } \\
\text { letzung }\end{array}$ & Repetitive Mikrotraumata durch Außenrotation und Spannungsüberlastung & $\begin{array}{l}\text { Komplette Risse der vorderen Kapsel, meist schräg } \\
\text { durch das vordere Band des Lig. glenohumeralis } \\
\text { inferior; Flüssigkeits- oder Kontrastmittelextravasa- } \\
\text { tion in die periartikulären Weichteile }\end{array}$ \\
\hline $\begin{array}{l}\text { M. subsca- } \\
\text { pularis }\end{array}$ & $\begin{array}{l}\text { Querausrichtung und Dehnung der unteren Subskapularisfasern bei Abdukti- } \\
\text { onsaußenrotation }\end{array}$ & $\begin{array}{l}\text { Flüssigkeitssignal und partielle/vollständige Dis- } \\
\text { kontinuität der unteren myotendinösen Verbin- } \\
\text { dung }\end{array}$ \\
\hline \multicolumn{3}{|c|}{ Verletzungen der hinteren Schulteranteile } \\
\hline $\begin{array}{l}\text { Bennet- } \\
\text { Läsion }\end{array}$ & $\begin{array}{l}\text { Wiederholte Traktion am hinteren Anteil des Lig. glenohumerale inferior am } \\
\text { Glenoidrand während der Dezeleration }\end{array}$ & $\begin{array}{l}\text { Bogenförmige Verknöcherung am posterior infe- } \\
\text { rioren Glenoidrand }\end{array}$ \\
\hline $\begin{array}{l}\text { Verletzungen } \\
\text { der hinteren } \\
\text { Muskeln }\end{array}$ & $\begin{array}{l}\text { M. latissimus dorsi bzw. M. teres major stabilisieren gegen vordere Anspan- } \\
\text { nungen beim „late cocking“, werden bei der Akzeleration maximal gespannt } \\
\text { und verkürzen sich beim "follow through“; M. infraspinatus und M. teres minor } \\
\text { wirken ableitend und erfahren bei der Dezeleration hohe Distraktionskräfte }\end{array}$ & $\begin{array}{l}\text { Fokales Ödem und Flüssigkeit am myotendinösen } \\
\text { Übergang des M. latissimus dorsi, M. teres major } \\
\text { bzw. M. infraspinatus }\end{array}$ \\
\hline
\end{tabular}

Schulterkomplexes kommt es außerdem zu langfristigen Schädigungen, die auch nach dem Ende der sportlichen Karriere zu Einschränkungen führen bzw. die normale Degeneration der Rotatorenmanschette beschleunigen $[25,28]$. Dabei sind nicht nur akute Verletzungen problematisch. Bereits bei $70 \%$ der Nachwuchsathleten sind morphologische Veränderungen der Schulter zu beobachten, obwohl es sich um asymptomatische Sportler handelt [3].

Die Höhe der Belastung wird stark durch die sportartspezifischen Anforderungen determiniert. Dabei spielen die Größe der Abwurfgeschwindigkeit, die Übertragung der Energie aus vorbereitenden Phasen auf das Wurfgerät sowie die optimale Koordination von Teilkörperbewegungen unter Ausnutzung der Trägheit von Körpersegmenten sowie zeitliche und räumliche Zwänge eine entscheidende Rolle $[2,19]$.

Die hohen und durch die Anzahl der Würfe stark repetitiven Belastungen führen zu einer Vielzahl von unterschiedlichen Verletzungs- und Beschwerdebildern.
- Tab. 1 führt die häufigsten überkopfsportspezifischen Schulterpathologien auf.

Obwohl über die Belastungs-/Beanspruchungssituation und die Verletzungen v.a. auch durch die Untersuchungen aus dem Baseball bereits relativ viele Ergebnisse vorliegen, ist unklar, welche Unterschiede durch die Spezifik anderer Schlagwurfbewegungen entstehen und welche Verletzungsmechanismen damit erklärt werden können. Ziel der Untersuchung war es deshalb, die vollständige Kinematik und Kinetik der Schulterbewegung im Speerwurf zu erfassen, um somit die Belastung der Schulter genauer zu beschreiben und Verletzungsrisiken zu identifizieren.

\section{Untersuchungsmethoden}

An den Untersuchungen nahmen 10 männliche Kaderathleten $(22,6 \pm 3,4$ Jahre; $1,88 \pm 0,07 \mathrm{~m} ; 92,8 \pm 10,8 \mathrm{~kg})$ des Deutschen Leichtathletikverbands teil. Alle Studienteilnehmer waren zum Zeitpunkt der Datenaufnahme frei von Verletzungen und gesundheitlichen Einschränkungen. Die Studie wurde in Übereinstimmung mit den Grundsätzen der Deklaration von Helsinki in ihrer aktuellen Fassung durchgeführt und von der lokalen Ethikkommission genehmigt (462/18-EK). Alle Teilnehmer wurden vor den Untersuchungen zu Risiken, Ablauf und Nutzen aufgeklärt und eine Einverständniserklärung eingeholt. Die Teilnahme an der Studie erfolgte freiwillig.

Nach einer individuellen Vorbereitung erfolgte die Präparation der Teilnehmer mit 18 reflektierenden Markern ( $\bullet$ Abb. 1). Die Hauptphase der Wurfbewegungen der Sportler wurden mit einem Infrarotkamerasystem (Qualisys AB, Göteborg, Schweden) bestehend aus 12 Infrarot- (Oqus 7+) und 2 Videokameras (Oqus 210c) erfasst. Die Kameras operierten dabei mit $250 \mathrm{~Hz}$ (infrarot) bzw. $125 \mathrm{~Hz}$ (Video). Die Sportler absolvierten im Anschluss mindestens 3 Würfe aus ihrem bevorzugten Anlauf, wobei das Augenmerk darauf lag, die höchstmögliche Abwurfgeschwindigkeit 


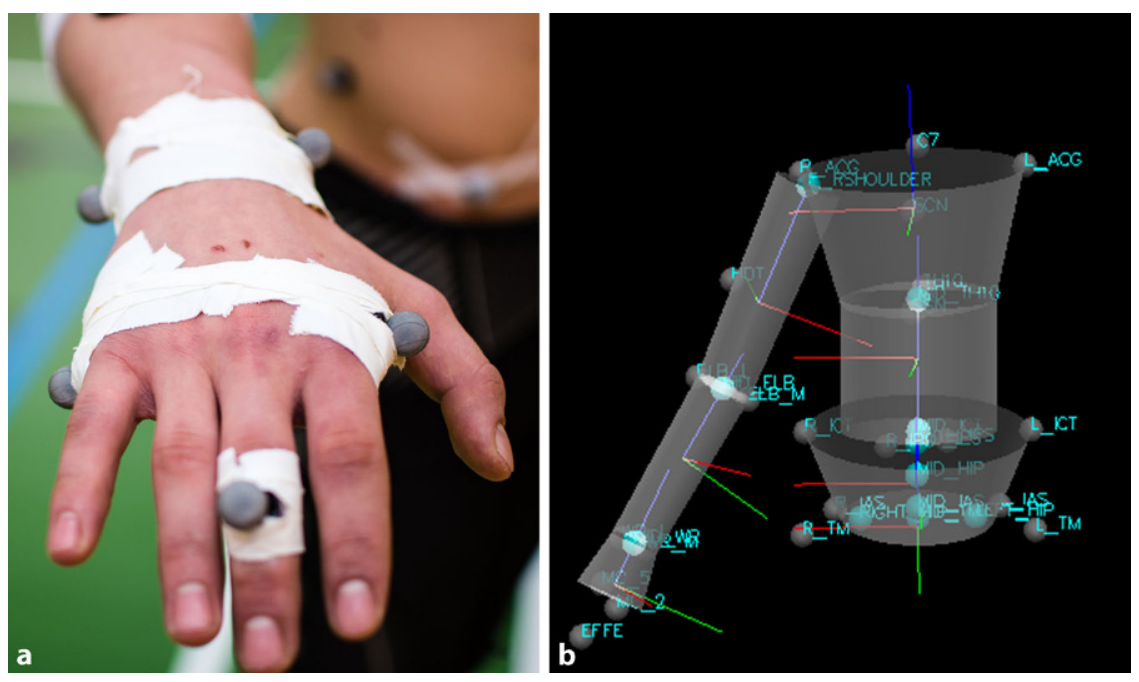

Abb. 1 ム Beispielhafte Bemarkerung der Hand mit zugehöriger Klebesicherung (a). Repräsentation des Körpers aus geometrischen Objekten (b)

$\left(v_{0}\right)$ des Speeres zu generieren. Aus den aufgezeichneten Versuchen eines Probanden wurde jeweils der Versuch mit der höchsten $v_{0}$ für die weitergehende Analyse ausgewählt. Nach einer vorhergehenden Residuenanalyse [29] erfolgte die Glättung der dreidimensionalen (3D-)Koordinaten jedes Markers mit den individuell optimalen Filterfrequenzen und einem Zerolag-Butterworth-Filter 4. Ordnung. Damit wurde anschließend ein Körpermodell in Anlehnung an Roach und Lieberman [22], bestehend aus der wurfarmseitigen Hand, Unterarm, Oberarm, Thorax und Abdomen gespeist, um die Kinematik der Gelenke zu berechnen. Hierfür konnte die Modellierungsumgebung Visual 3D (C-Motion Inc., Germantown, MD, USA) genutzt werden. Mit Hilfe der Kinematik und den Trägheitsdaten der Segmente von De Leva [16] erfolgte im Anschluss die Berechnung der Gelenkkinetik unter Zuhilfenahme der Newton-Euler-Bewegungsgleichungen. Alle Kräfte und Momente wurden als interne Kräfte/Momente berechnet. Um eine bessere Interpretierbarkeit der Daten zu gewährleisten, wurden alle Daten in ein Gelenkkoordinatensystem transformiert [12].

Aus den so berechneten Verläufen erfolgte die Extraktion der maximalen Momente und Winkelgeschwindigkeiten für die Bewegungsrichtungen der Innenrotation (Ir), der horizontalen Flexion (hFlex) und der Abduktion (Abd). Zusätzlich dazu wurden die Bewegungsamplituden aufder
Basis der maximal erreichten Gelenkwinkel in der Außenrotation (Ar) und horizontalen Extension (hExt) parametrisiert. Da in der Bewegungsebene der Add-/Abduktion keine maximalen Amplituden erreicht werden, erfolgte nur die Erfassung der kleinsten Ausprägung. Um weiterhin Aussagen über die perspektivischen Anforderungen bei höheren Abwurfgeschwindigkeiten zu bestimmen bzw. den Zusammenhang mit der Abwurfgeschwindigkeit zu ermitteln, wurden für die Winkel, Winkelgeschwindigkeiten und die Gelenkmomente der Zusammenhang zur Abwurfgeschwindigkeit in Form einer Korrelationsanalyse nach Pearson geprüft und im Fall eines signifikanten Zusammenhangs eine Regressionsanalyse durchgeführt. Für die Korrelation wurde zusätzlich das $95 \%$-Konfidenzintervall (95\%-KI) berechnet. Da die Gelenkmomente im großen Maß durch die Körpermaße (KM) und die Körperhöhe (KH) beeinflusst sind, wurde die Korrelations- und Regressionsanalyse für Momente durchgeführt, die anhand des Produkts aus $\mathrm{K}$ und $\mathrm{KM}$ normalisiert wurden [20]. Das Signifikanzniveau wurde vorab auf $a=0,05$ festgesetzt.

\section{Ergebnisse}

Im Mittel erreichten die Sportler eine Abwurfgeschwindigkeit $\left(\mathrm{v}_{0}\right)$ von $23,29 \pm$ 2,17 $\mathrm{ms}^{-1}$. $\bullet$ Tab. 2 zeigt die maximal ermittelten Gelenkwinkelamplituden. Für diese konnten keine Zusammenhänge zur $\mathrm{v}_{0}$ ermittelt werden. Die ermittelten Gelenkwinkelgeschwindigkeiten bewegen sich zwischen $4071,47 \pm 360,65 \%$ und $445,01 \pm 96,77 / \mathrm{s}$, abhängig von der Bewegungsebene, wobei sich auch innerhalb der Bewegungsebenen z. T. deutliche Unterschiede ergeben (- Tab. 3). Weiterhin konnte für die ermittelte maximale Innenrotationsgeschwindigkeit ein signifikanter Zusammenhang zur Abwurfgeschwindigkeit ermittelt werden: Mit zunehmender Abwurfgeschwindigkeit nimmt auch die maximale Innenrotationgeschwindigkeit zu. Beispielhafte Verläufe der Winkelgeschwindigkeiten der unterschiedlichen Bewegungsebenen zeigt $\square$ Abb. 2a. Während die maximalen Winkelgeschwindigkeiten der Abduktion und der horizontalen Flexion noch vor dem Abwurf erreicht werden, steigt die Winkelgeschwindigkeit in der Innenrotation bis zum Abwurf weiter an, das Maximum wird erst nach dem Abwurf erreicht.

Für die auftretenden Gelenkmomente zeigen sich ebenfalls Unterschiede zwischen den Bewegungsebenen (- Tab. 4). - Abb. 2b zeigt beispielhaft den Verlauf der Gelenkmomente in den unterschiedlichen Bewegungsebenen, wobei zusätzlich die entsprechenden Kontraktionsformen der Muskulatur eingezeichnet sind. Für das maximale Innenrotationsmoment konnte weiterhin ein Zusammenhang zur Abwurfgeschwindigkeit ermittelt werden: Mit steigender Abwurfgeschwindigkeit steigt auch das Innenrotationsmoment. Die maximalen Momente in den unterschiedlichen Bewegungsrichtungen werden jeweils am Bewegungsumkehrpunkt erreicht, wobei bis zu diesem Zeitpunkt das Gelenkmoment durch eine exzentrische Arbeitsweise aufgebracht wird (• Abb. 3).

\section{Diskussion}

Mit der in der vorliegenden Untersuchung erreichten Abwurfgeschwindigkeit ordnen sich die Sportler unterhalb von Wettkampfanalysen ein. Die Auswertungen verschiedener Weltmeisterschaften haben gezeigt, dass im Hochleistungsbereich Abwurfgeschwindigkeiten von $>30 \mathrm{~ms}^{-1}$ erreicht werden können [15, 19]. Noch höhere Abwurfgeschwindigkeiten $\left(40 \mathrm{~ms}^{-1}\right)$ werden im Baseball erreicht [6]. Entsprechende Unterschiede lassen 
Tab. 2 Mittelwerte $(x)$ und Standardabweichung $(s)$ der maximal erreichten Gelenkamplituden in den unterschiedlichen Bewegungsrichtungen. Zusätzlich angegeben sind Min- und Maxima ( $\min ;$ max), sowie die Korrelation mit Abwurfgeschwindigkeit inklusive $95 \%$-Konfidenzintervall $(95 \%-K I)$ und dem Signifikanzwert $(p)$

\begin{tabular}{|l|l|l|l|l|l|}
\hline$\circ$ & $\mathbf{x}$ & $\mathbf{s}$ & Min; $\max$ & $\mathbf{r}(\mathbf{9 5 \% - K I )}$ & $p$ \\
\hline Ar & 143,35 & $\pm 9,56$ & 128,$27 ; 155,41$ & $0,284(-0,775 ; 0,421)$ & 0,425 \\
\hline hExt & 26,41 & $\pm 7,92$ & 6,$53 ; 36,36$ & $0,313(-0,394 ; 0,787)$ & 0,378 \\
\hline Abd & 74,86 & $\pm 9,70$ & 95,$38 ; 64,78$ & $-0,132(-0,703 ; 0,542)$ & 0,714 \\
\hline \multicolumn{7}{|l}{ Ar Außenrotation, $h$ Ext horizontale Extension, Abd Abduktion } \\
\hline
\end{tabular}

Tab. 3 Mittelwerte $(x)$ und Standardabweichung $(s)$ der maximal erreichten Gelenkwinkelgeschwindigkeiten in den unterschiedlichen Bewegungsrichtungen. Zusätzlich angegeben sind Min-und Maxima ( $\min$; $\max$ ) sowie die Korrelation mit der Abwurfgeschwindigkeit inklusive $95 \%$ Konfidenzintervall (95\%-KI) und dem Signifikanzwert $(p)$

\begin{tabular}{|c|c|c|c|c|c|c|c|}
\hline$\% / \mathrm{s}$ & $x$ & $s$ & Min; $\max$ & r (95\%-KI) & $p$ & $\mathrm{~m}^{\mathrm{a}}$ & $\mathbf{b}^{\mathbf{a}}$ \\
\hline $\mathrm{Ir}$ & 4071,47 & $\pm 360,65$ & 3605,$9 ; 4647,2$ & $0,707(0,141 ; 0,925)$ & 0,022 & 117,71 & 1255,80 \\
\hline hFlex & 445,01 & $\pm 96,77$ & 274,$53 ; 611,03$ & $0,481(-0,213 ; 0,853)$ & 0,159 & - & - \\
\hline Abd & 504,14 & $\pm 153,37$ & 173,$63 ; 691,77$ & $0,221(-0,475 ; 0,747)$ & 0,539 & - & - \\
\hline
\end{tabular}

Tab. 4 Mittelwerte $(x)$ und Standardabweichung $(s)$ der maximal erreichten Gelenkmomente in den unterschiedlichen Bewegungsrichtungen.Zusätzlich angegeben sind Min-und Maxima ( $m i n$; max), sowie die Korrelation mit der Abwurfgeschwindigkeit inklusive $95 \%$-Konfidenzintervall $(95 \%-K I)$ und dem Signifikanzwert $(p)$

\begin{tabular}{|c|c|c|c|c|c|c|c|}
\hline $\mathrm{Nm}$ & $x$ & $s$ & Min; max & r (95\%-KI) & $p$ & $\mathrm{~m}^{\mathrm{a}}$ & $\mathbf{b}^{\mathbf{a}}$ \\
\hline Ir & 112,52 & $\pm 25,27$ & 69,$53 ; 161,76$ & $0,710(0,144 ; 0,926)$ & 0,021 & 0,028 & $-0,054$ \\
\hline hFlex & 129,63 & $\pm 24,15$ & 95,$60 ; 170,94$ & $0,425(-0,279 ; 0,832)$ & 0,220 & - & - \\
\hline Abd & 109,63 & $\pm 21,53$ & 84,$91 ; 150,87$ & $0,378(-0,330 ; 0,814)$ & 0,282 & - & - \\
\hline
\end{tabular}

sich auch in den Ausprägungen der Winkel und Winkelgeschwindigkeiten finden. Während die horizontale Extension im Baseball einen ähnlichen Wert annimmt (im Mittel $21 \pm 12^{\circ}-27 \pm 10^{\circ}$ ), zeigen sich deutliche Unterschiede in der Außenrotation. Hier wurden in der Vergangenheit Werte bis zu $185 \pm 10^{\circ}$ berichtet [8]. Während sich die horizontale Flexion noch innerhalb der Beweglichkeitsgrenzen (40-50 bewegt, ist die Bewegungsamplitude in der Außenrotation auf das Doppelte der Bewegungsreichweite nach Neutral-NullMethode $\left(70^{\circ}\right)$ erhöht [24].

Auch die Winkelgeschwindigkeiten zeigen sich gegenüber den Werten aus dem Baseball verringert. Während den Autoren im Baseball keine Werte zur horizontalen Flexion bekannt sind, wurden in der Innenrotation Werte von bis zu $8000 \%$ serichtet [8]. Obwohl sich die bisherigen Werte vermindert gegenüber dem Baseball gezeigt haben, liegt die Gelenkbelastung da- rüber. Für die horizontale Flexion werden im Baseball mittlere Werte bis $110 \mathrm{Nm}$ berichtet, für die Innenrotation bis $98 \mathrm{Nm}[9$, 11].

Die Unterschiede in den Ausprägungen der Merkmale sind dabei auf unterschiedliche Faktoren zurückzuführen. Zum einen spielt die Gerätemasse und Form eine entscheidende Rolle. Der Speer ist mit seinen rund $\mathrm{ca} .800 \mathrm{~g}$ deutlich schwerer als ein Baseball (ca. $145 \mathrm{~g}$ ) und besitzt durch seine Länge von ca. 2,6 m zusätzlich ein verändertes Massenträgheitsmoment. Zum anderen ist zu bedenken, dass die Speerwerfer durch den Anlauf eine höhere Eingangs- bzw. Anfangsgeschwindigkeit besitzen als die Baseballspieler. Durch die vergrößerte Trägheit, verbunden mit den technischen Anforderungen, die Teilkörperbewegungen nacheinander zu initiieren, verbleibt der Arm samt Gerät hinter dem Oberkörper zurück, wodurch der Arm in eine Stellung aus Außenrotation und horizontaler Extension gedrückt wird [23]. Durch die größere Masse des Geräts werden jedoch deutlich höhere Anforderungen an die Sicherung des Schultergelenks gestellt, wie sich in den erhöhten Gelenkmomenten zeigt, gleichzeitig vermindern die verstärkten Sicherungsmechanismen durch eine Erhöhung der Muskelspannung eine höhere Außenrotation, wie sie im Baseball zu finden ist.

Es ist jedoch gleichzeitig zu erwarten, dass durch die gefundenen Zusammenhänge zwischen dem Innenrotationsmoment und der Abwurfgeschwindigkeit eine weitere Erhöhung der Gelenkmomente im Wettkampf zu erwarten ist. Für einen 100kg-Sportler von 1,9 m Körpergröße, der eine Abwurfgeschwindigkeit von $30 \mathrm{~ms}^{-1} \mathrm{er}-$ reicht (ca. $90 \mathrm{~m}$ ), sind ca. $150^{\circ} \mathrm{Nm}$ an Belastung erwartbar. Aus Leistungssicht sind somit die gestellten Anforderungen aus extremen Gelenkamplituden und zugehörigen hohen Gelenkmomenten, auch als "throwers paradox" bekannt, unerlässlich [28].

Wie neuere Untersuchungen aus dem Baseball zeigen, trägt die Phase der exzentrischen Außenrotation maßgeblich zur Beschleunigung des Geräts bei [1]. Eine verminderte Schulterstabilität ist somit nicht nur ein Gefahrenpotenzial für Verletzungen, sondern darüber hinaus auch leistungslimitierend. Die Sportler müssen daher im Training dazu befähigt werden, bei einer extremen Außenrotation hohe Kräfte zu generieren, wodurch zum einen ein hohes Maß an Energietransfer sichergestellt wird und zum anderen die Stabilität des Gelenks gewährleistet werden kann [14]. Dabei ist jedoch zu beachten, dass die Muskulatur einer exzentrischen Arbeitsweise unterliegt.

Neben den Anforderungen in der Beschleunigungsphase werden auch an das Abbremsen der Wurfbewegungen extreme Anforderungen gestellt. Die erreichten Innenrotationsgeschwindigkeiten müssen im Zug der Bremsphase abgefangen werden. Auch hierbei ist die vorrangige Arbeitsweise exzentrisch. Es ist zu erwarten, dass mit steigender Leistungsfähigkeit auch höhere Winkelgeschwindigkeiten erreicht werden, wie es die Zusammenhangsanalyse gezeigt hat. Es muss somit genügend Weg zur Verfügung stehen, um die Winkelgeschwindigkeit zu verringern, 

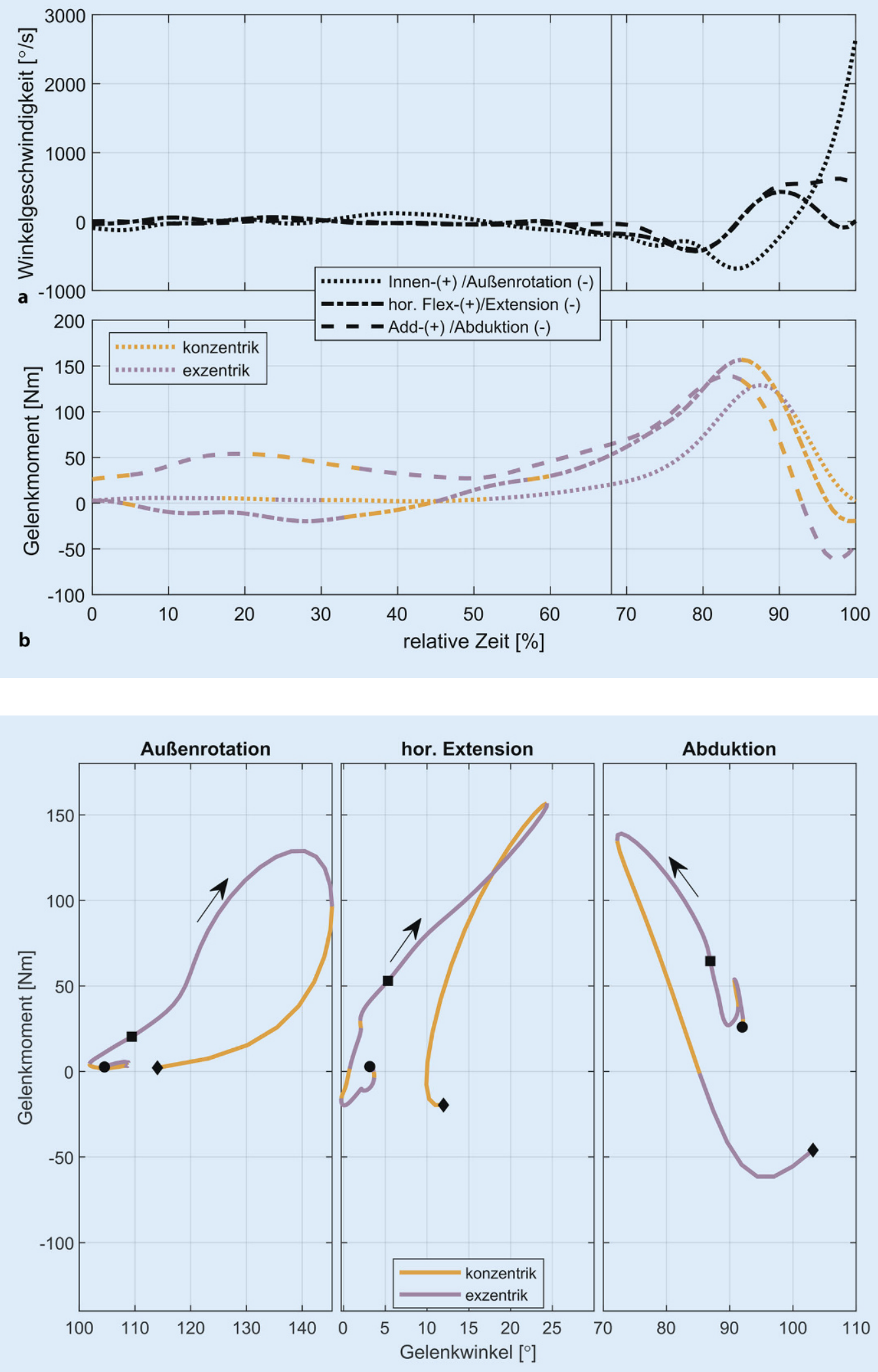

Abb. $2 \triangleleft$ Zeitnormalisierte WinkelgeschwindigkeitsZeit-Verläufe (a) und Moment-Zeit-Verläufe (b) für die unterschiedlichen Bewegungen der Schulter. Im unteren Diagramm ist zusätzlich die Kontraktionsform der Muskulatur gekennzeichnet
Abb. $3 \triangleleft$ Winkel-MomentDiagramme für die drei Bewegungen im Schultergelenk. Farblich gekennzeichnet sind die Kontraktionsformen, die Pfeile geben die zeitliche Richtung der Werte an. Durch die Symbole werden der Beginn der Hauptphase (O), der Aufsatzzeitpunkt des Stemmbeins ( $\square$ ) und der Abwurfzeitpunkt $(\diamond)$ markiert 
da sonst entweder die Anforderungen an die abfangende Muskulatur steigen oder bei deren verminderter Leistungsfähigkeit ein Abfangen über die passiven Strukturen erfolgt.

Während der Abbremsbewegung treten insgesamt die größten Kräfte im Schultergelenk auf. Durch exzentrische Kontraktion der Schultermuskeln, besonders der Rotatorenmanschette, des M. deltoideus und der Rumpfmuskulatur, stehen diese unter enormem Dehnungsstress. Diese Erkenntnis ist insbesondere für Überkopfsportler mit Innenrotationsdefizit relevant. Das sog. glenohumerale Innenrotationsdefizit (GIRD) wird auf eine Kontraktur der dorsalen muskulokapsulären Weichteile und mit einer daraus folgenden Verschiebung, dem glenohumeralen Kontaktpunkt, nach posterokranial zurückgeführt [4]. Die adaptiven Vorgänge verlaufen meist asymptomatisch und bleiben primär naturgemäß unbehandelt. Das "totale ROM concept" beschreibt hierbei einen physiologischen Zustand für den individuellen Sportler bei seitengleichem Gesamtausmaß der Rotationsfähigkeit. Seitendifferenzen $>20^{\circ}$ im Rotationsgesamtsektor werden als pathologisch und somit behandlungsbedürftig angesehen [21].

Vor dem Hintergrund der hier dargestellten Ergebnisse scheint die einwirkende Kraft auf den verkürzten Bremsweg einen relevanten Einfluss auf die werferschulterspezifischen Pathologien zu haben, wobei der Übergang einer physiologisch adaptierten Werferschulter zu einer therapiebedürftigen pathologischen Werferschulter meist fließend ist.

\section{Limitationen}

Die Untersuchungen an den Sportlern wurden 2 Monate vor Beginn der Wettkampfsaison durchgeführt, die Abwurfgeschwindigkeiten, die Bewegungsstruktur und auch die Belastungssituation sind somit mit Annäherung an den Wettkampfhöhepunkt noch Veränderungen unterworfen. Es ist davon auszugehen, dass sich v. a. die Belastungssituation während der fortschreitenden Saison nochmals verändert.

Weiterhin müssen für die Modellierung der Belastung Annahmen getroffen wer- den, die v. a. die berechneten Belastungen beeinflussen können. So werden Weichteilbewegungen nicht berücksichtigt, da die Körpersegmente als starre Körper angenommen werden. Die verwendeten Trägheitseigenschaften der Segmente sind zusätzlich als Fehlerpotenzial zu betrachten, da hier nicht die individuellen anthropometrischen Eigenschaften der Sportler berücksichtigt werden und es sich somit um eher generalisierte Werte handelt [27].

\section{Fazit für die Praxis}

- Die vorliegende Studie ermittelte erstmals die Belastungssituation der Schulterstrukturen im Speerwurf.

- Es konnte gezeigt werden, dass sich trotz im Vergleich zum Baseball geringerer Abwurfgeschwindigkeiten die Belastung der Schulterstrukturen z. T. deutlich erhöht. Die auftretenden Verletzungsmechanismen sind dabei gleichartig: hohe Gelenkamplituden, verbunden mit extremen Gelenkmomenten stellen ein hohes Risiko für Verletzungen dar, wobei auch die Anforderungen an die Bremsphase der Bewegung enorme Belastungen für die Schulterstrukturen darstellen.

- Eine gründliche und systematische Vorbereitung von Wurfbelastungen im Trainingsprozess ist daher unabdingbar. Dabei sollten v. a. die spezifischen Belastungsnormative hinsichtlich der Bewegungsamplitude, der Kraftanforderungen und der Kontraktionsform, derer die Muskulatur in den entscheidenden Phasen unterliegt, berücksichtigt werden.

- Bereits bestehende Vorbereitungsprogramme sollten vor diesem Hintergrund kritisch hinterfragt und entsprechend angepasst werden.

\section{Korrespondenzadresse}

\section{Dr. Hans-Peter Köhler}

Sportwissenschaftliche Fakultät, Abteilung Sportbiomechanik, Universität Leipzig Jahnallee 59, 04109 Leipzig, Deutschland hans-peter.koehler@uni-leipzig.de

Funding. Open Access funding enabled and organized by Projekt DEAL.

\section{Einhaltung ethischer Richtlinien}

Interessenkonflikt. H.-P. Köhler, P. Hepp und M. Witt geben an, dass kein Interessenkonflikt besteht.

Alle beschriebenen Untersuchungen am Menschen oder an menschlichem Gewebe wurden mit Zustim- mung der zuständigen Ethikkommission, im Einklang mit nationalem Recht sowie gemäß der Deklaration von Helsinki von 1975 (in der aktuellen, überarbeiteten Fassung) durchgeführt. Von allen beteiligten Patienten liegt eine Einverständniserklärung vor.

Open Access. Dieser Artikel wird unter der Creative Commons Namensnennung 4.0 International Lizenz veröffentlicht, welche die Nutzung, Vervielfältigung, Bearbeitung, Verbreitung und Wiedergabe in jeglichem Medium und Format erlaubt, sofern Sie den/die ursprünglichen Autor(en) und die Quelle ordnungsgemäß nennen, einen Link zur Creative Commons Lizenz beifügen und angeben, ob Änderungen vorgenommen wurden.

Die in diesem Artikel enthaltenen Bilder und sonstiges Drittmaterial unterliegen ebenfalls der genannten Creative Commons Lizenz, sofern sich aus der Abbildungslegende nichts anderes ergibt. Sofern das betreffende Material nicht unter der genannten Creative Commons Lizenz steht und die betreffende Handlung nicht nach gesetzlichen Vorschriften erlaubt ist, ist für die oben aufgeführten Weiterverwendungen des Materials die Einwilligung des jeweiligen Rechteinhabers einzuholen.

Weitere Details zur Lizenz entnehmen Sie bitte der Lizenzinformation auf http://creativecommons.org/ licenses/by/4.0/deed.de.

\section{Literatur}

1. Alderink GJ, Kepple T, Stanhope SJ, Aguinaldo A (2021) Upper body contributions to pitched ball velocity in elite high school pitchers using an induced velocity analysis. J Biomech 120:110360. https://doi.org/10.1016/j.jbiomech.2021.110360

2. Bartonietz K (2000) Javelin throwing: an approach to performance development. In: Zatsiorsky VM (Hrsg) Biomechanics in sport: performance enhancement and injury prevention. Blackwell Science, Oxford, S401-434

3. Beitzel K, Zandt JF, Buchmann S et al (2016) Structural and biomechanical changes in shoulders of junior javelin throwers: a comprehensive evaluation as a proof of concept for a preventive exercise protocol. Knee Surg Sports Traumatol Arthrosc 24:1931-1942. https://doi.org/10.1007/ s00167-014-3223-y

4. Burkhart SS, Morgan CD, Ben Kibler W (2003) The disabled throwing shoulder: spectrum of pathology part I: pathoanatomy and biomechanics. Arthroscopy 19:404-420. https://doi.org/10. 1053/jars.2003.50128

5. D'Souza D (1994) Track and field athletics injuries-a one-year survey. Br J Sports Med 28:197-202. https://doi.org/10.1136/bjsm.28.3. 197

6. Dowling B, Laughlin WA, Gurchiek RD et al (2020) Kinematic and kinetic comparison between American and Japanese collegiate pitchers. J Sci Med Sport 23:1202-1207. https://doi.org/10. 1016/j.jsams.2020.04.013

7. Edouard P, Depiesse F, Serra J-M (2010) Throwing arm injuries in high-level athletics throwers. Sci Sports 25:318-322. https://doi.org/10.1016/j. scispo.2010.08.004

8. Escamilla RF, Moorman C, Fleisig G et al (2002) Baseball: kinematic and kinetic comparisons between American and Korean professional baseball pitchers. Sports Biomech 1:213-228. https://doi.org/10.1080/14763140208522798 
9. Escamilla RF, Slowik JS, Diffendaffer AZ, Fleisig GS (2018) Differences among overhand, 3-quarter, and sidearm pitching biomechanics in professional baseball players. J Appl Biomech 34:377-385. https://doi.org/10.1123/jab.2017-0211

10. Fares MY, Fares J, Baydoun H, Fares Y (2020) Prevalence and patterns of shoulder injuries in Major League Baseball. Phys Sportsmed 48:63-67. https://doi.org/10.1080/00913847.2019.1629705

11. Feltner ME, Dapena J (1986) Dynamics of the shoulder and elbow joints of the throwing arm during a baseball pitch. Int J Sport Biomech 2:235-259. https://doi.org/10.1123/ijsb.2.4.235

12. Fleisig GS, Andrews JR, Dillman CJ, Escamilla RF (1995) Kinetics of baseball pitching with implications about injury mechanisms. Am J Sports Med 23:233-239. https://doi.org/10.1177/ 036354659502300218

13. Hepp P, Henkelmann R (2020) Die „HandballerSchulter" im Fokus von Diagnostik und Therapie. Sportverletz Sportschaden 34:153-162. https:// doi.org/10.1055/a-1107-8514

14. Köhler H-P (2020) Untersuchung zur Quantifizierung des Energieeintrags und -transfers innerhalb der oberen Extremitäten und deren Bedeutung für die Wettkampfleistung im Speerwurf der Männer

15. Lehmann F, Badura M, Schaa W, Perlt B (2010) Kennzeichnung sporttechnischer Entwicklungstendenzen in den leichtathletischen Wurfdisziplinen sowie im Stabhochsprung anhand der Analyse der Leichtathletik-WM 2009. Z Angew Trainingswiss 17:46-74

16. de Leva P (1996) Adjustments to Zatsiorsky-Seluyanov's segment inertia parameters. J Biomech 29:1223-1230

17. Lin DJ, Wong TT, Kazam JK (2018) Shoulder injuries in the overhead-throwing athlete: epidemiology, mechanisms of injury, and imaging findings. Radiology 286:370-387. https://doi.org/10.1148/ radiol.2017170481

18. Luig P (2015) Verletzungen im deutschen Profihandball der Männer - Epidemiologische Aspekte von Wettkampfverletzungen bei Erst-und Zweitligaspielern (2010-2013) unter Berücksichtigung systematischer Videoanalyen. Universität Bochum,

19. Morriss C, BartlettR, FowlerN (1997) Biomechanical analysis of the men's javelin throw at the 1995 world championships in athletics. New Stud Athl 12:31-41

20. Oi T, Slowik JS, Diffendaffer AZ et al (2019) Biomechanical differences between Japanese and American professional baseball pitchers. Orthop J Sports Med. https://doi.org/10.1177/ 2325967119825625

21. Pieper H-G, Muschol M (2014) Die Schulter des Wurf- und Überkopfsportlers. Sport Orthop Traumatol 30:19-24. https://doi.org/10.1016/j. orthtr.2013.11.002

22. Roach NT, Lieberman DE (2014) Upper body contributions to power generation during rapid, overhand throwing in humans. J Exp Biol 217:2139-2149. https://doi.org/10.1242/jeb. 103275

23. Roach NT, Venkadesan M, Rainbow MJ, Lieberman DE (2013) Elastic energy storage in the shoulder and the evolution of high-speed throwing in Homo. Nature 498:483-486. https://doi org/10.1038/nature 12267

24. Ryf C, Weymann A (1995) The neutral zero method-a principle of measuring joint function. Injury 26:1-11. https://doi.org/10.1016/00201383(95) $90116-7$

\section{Load quantification on the muscle-ligament apparatus in the javelin} thrower's shoulder

Background: Shoulder injuries are common causes for loss of training time and inability to compete. However, not only acute injuries are problematic, but also longterm changes of the stabilizing structures. While some studies are already available on the loading situation of the shoulder in baseball, this is not yet the case in javelin throwing, neither the kinematics nor the kinetics have been fully quantified so far. Objective: The aim of the work was to quantify the load on the shoulder in the javelin throw, and thus contribute to the identification of injury risks.

Materials and methods: The javelin throwing motions of 10 male athletes were recorded using an infrared camera system and 18 retro-reflective markers. These data were used in a 5-segmental multibody model to calculate the kinematics and kinetics of the shoulder joint. The peak values were extracted from the different kinematic and kinetic time series and correlated with release speed.

Results: Athletes achieved a mean release velocity of $23.29 \pm 2.17 \mathrm{~ms}^{-1}$. Maximum joint angular velocities ranged from $445-4071 \%$ depending on the plane of motion. There were also plane-specific differences in joint loads, which ranged from 109-129 Nm. Conclusion: Compared to baseball, the load situation in the javelin throw is different. While the javelin throw shows reduced amplitudes and angular velocities, an increased force requirement is visible. The reasons for this may be found in the different sets of rules (device weight/dimensions, input velocity).

\section{Keywords}

Rotator cuff · Dynamics · Modelling · Simulation · Performance sports

25. Schmitt H, Hansmann HJ, Brocai DRC, Loew M (2001) Long term changes of the throwing arm of former elite javelin throwers. Int J Sports Med 22:275-279. https://doi.org/10.1055/s-200113814

26. Shanley E, Kissenberth MJ, Thigpen CA et al (2015) Preseason shoulder range of motion screening as a predictor of injury among youth and adolescent baseball pitchers. JShoulderElbow Surg 24:1005-1013. https://doi.org/10.1016/j.jse. 2015.03.012

27. Sterner JA, Reaves SK, Aguinaldo AL et al (2020) Inverse dynamics analysis of youth pitching arm kinetics using body composition imaging. Sports Biomech. https://doi.org/10.1080/14763141. 2020.1715470

28. Wilk KE, Meister K, Andrews JR (2002) Current concepts in the rehabilitation of the overhead throwing athlete. Am J Sports Med 30:136-151. https://doi.org/10.1177/03635465020300011201

29. Winter DA (2009) Biomechanics and motor control of human movement, 4 . Aufl. John Wiley \& Sons, Hoboken, New Jersey 